\title{
Effect of Different Sources and Levels of Sulphur on Growth and Yield of Maize (Zea mays L.)
}

\author{
P.V. Padma", G.E.CH. Vidyasagar, P. Suresh and S. Harish Kumar Sharma \\ Department of Agronomy, College of Agriculture, Professor Jayashankar Telangana State \\ Agricultural University, Hyderabad-030, Telangana, India \\ *Corresponding author
}

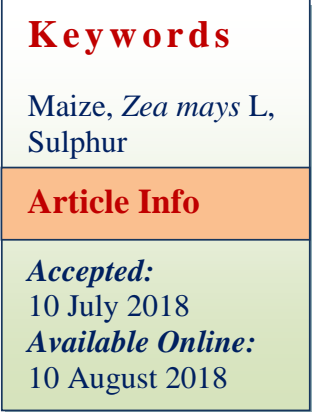

\section{A B S T R A C T}

A field experiment was conducted during Kharif, 2017 at College Farm, College of Agriculture, Rajendranagar, Hyderabad to compute the optimum dose and source of sulphur in maize and to evaluate the impact of sulphur on growth, yield attributes and yield of maize. The experiment was carried out with three different sources of sulphur (Gypsum, Ammonium Sulphate and Bentonite Sulphur) and three levels (S@ $40 \mathrm{~kg} \mathrm{ha}^{-1}, \mathrm{~S} @ 60 \mathrm{~kg}$ $\mathrm{ha}^{-1}$ and S @80 $\mathrm{kg} \mathrm{ha}^{-1}$ ) and one source as urea, DAP and MOP and another source as urea, SSP and MOP comprising of eleven treatment combinations which were laid out in randomized block design and replicated thrice. In all the treatments nitrogen was applied in two split doses 50\% as basal and 50\% as top dressing at 45 DAS and entire dose of phosphorous, potassium and sulphur were applied as basal. Treatment $\mathrm{T}_{1}$ was application of recommended dose of fertilizer i.e., N- $\mathrm{P}_{2} \mathrm{O}_{5}-\mathrm{K}_{2} \mathrm{O}-\mathrm{S} @ 200-60-50-60 \mathrm{~kg} \mathrm{ha}^{-1}$ without sulphur. Treatment $T_{2}$ was application of recommended dose of fertilizer through Urea, SSP and MOP. $\mathrm{T}_{3}$ was $\mathrm{T}_{1}+$ application of sulphur @ $40 \mathrm{~kg} \mathrm{ha}^{-1}$ through gypsum; $\mathrm{T}_{4}$ was $\mathrm{T}_{1}+$ application of sulphur @ $60 \mathrm{~kg} \mathrm{ha}^{-1}$ through gypsum; $\mathrm{T}_{5}$ was $\mathrm{T}_{1}+$ application of sulphur @80 kg ha ${ }^{-1}$ through gypsum. $\mathrm{T}_{6}$ was $\mathrm{T}_{1}+$ application of sulphur @ $40 \mathrm{~kg} \mathrm{ha}^{-1}$ through ammonium sulphate. $\mathrm{T}_{7}$ was $\mathrm{T}_{1}+$ application of sulphur @ $60 \mathrm{~kg} \mathrm{ha}^{-1}$ through ammonium sulphate. $\mathrm{T}_{8}$ was $\mathrm{T}_{1}+$ application of sulphur @ $80 \mathrm{~kg} \mathrm{ha}^{-1}$ through ammonium sulphate. $\mathrm{T}_{9}$ was $\mathrm{T}_{1}+$ application of sulphur @ $40 \mathrm{~kg} \mathrm{ha}^{-1}$ through bentonite sulphur. $\mathrm{T}_{10}$ was $\mathrm{T}_{1}+$ application of sulphur @ $60 \mathrm{~kg} \mathrm{ha}^{-1}$ through bentonite sulphur. $\mathrm{T}_{10}$ was $\mathrm{T}_{1}+$ application of sulphur @ $80 \mathrm{~kg} \mathrm{ha}^{-1}$ through bentonite sulphur. Highest grain $(5596 \mathrm{~kg}$ $\left.\mathrm{ha}^{-1}\right)$ and stover yield $\left(6995 \mathrm{~kg} \mathrm{ha}^{-1}\right)$ was recorded by treatment $\mathrm{T}_{10}\left(\mathrm{~S} @ 60 \mathrm{~kg} \mathrm{ha}^{-1}\right.$ through bentonite sulphur) followed by treatment $\mathrm{T}_{2}\left(5581\right.$ and $\left.6976 \mathrm{~kg} \mathrm{ha}^{-1}\right) \mathrm{S} @ 40 \mathrm{~kg} \mathrm{ha}^{-1}$ through urea, SSP and DAP. Highest gross returns were recorded by $\mathrm{T}_{10}$ (Rs. $80139 \mathrm{ha}^{-1}$ ) followed by $T_{2}\left(\right.$ Rs. 78623 ha $^{-1}$ ) though highest $\mathrm{B}$ : C ratio was recorded by $\mathrm{T}_{2}$ because of higher cost of bentonite sulphur fertilizer.

\section{Introduction}

Maize is one of the important cereal crops in the world's agricultural economy both as food for humans and feed for animals, because of its higher yield potential compared to other cereals it is called as "Queen of Cereals". Maize is a miracle crop as there is no cereal crop on earth which has so immense potentiality and nutrient extensive feature. 
India is ranks $6^{\text {th }}$ in World's maize production. In India, maize is cultivated in an area of 8.85 lakh ha with a total production of 22.84 lakh tons and productivity of $2580 \mathrm{~kg} \mathrm{ha}^{-1}$ (Department of Economics and Statistics, 2017). Maize has been considered as highly nutrient responsive crop with wide adaptability and compatibility under diverse soil and climatic conditions. It has a variety of uses and has become popular due to its nutritive value and wide spread acceptability by humans. In India, maize (Zea mays L.) is the third most important cereal after rice and wheat that provides food, feed, fodder and serves as a source of raw material for developing hundreds of industrial products viz., starch, protein, oil, alcoholic beverages, food sweeteners, pharma, cosmetics, bio-fuel etc. Maize contains $4.5 \%$ oil which is ideal cooking medium for various recipes. About $66 \%$ of total maize production is used as feed, $25 \%$ as food and industrial products while the rest is used as seed etc. (Binod kumar et al., 2016). Due to higher yield potential, short growing period, high value food, forage and feed for livestock, poultry and a cheaper source of raw material for agro based industry, it is increasingly gaining importance in cropping systems. Among many reasons for low productivity, mismanagement of plant nutrition and agronomic practices are considered to be the major ones. Hence, for getting higher maize production of better quality, there is a need to improve these two major components of production technology.

Sulphur is one of the 16 elements essential for crop production (Marshner, 1995). Sulphur is considered as the fourth major nutrient element for crops (Platou and Jones, 1982). It is typically considered a secondary macronutrient (along with calcium and magnesium), but is essential for maximum crop yield and quality. Sulphur is often ranked immediately behind nitrogen, phosphorus and potassium in terms of importance to crop productivity (Krishnamoorthy, 1989 and Patil 1998). Sulphur is a component of the amino acids cysteine, cystine and methionine (Gangadhara et al., 1990 and Kumar and Yadav, 2007) making it essential for protein synthesis in plants. Plants also contain a large variety of other organic sulphur compounds such as glutathione. Sulphur is also a constituent of vitamins (thiamine and biotin), glycosides and co-enzyme A (Tisdale et al., 1985).

In recent years sulphur deficiency has become an increasing problem in agriculture. Saalbach (1973) reported maize yield loss to an extent of 10 to $30 \%$ and Pal and Singh (1992) upto $35 \%$ due to Sulphur deficiency. In general, cereals have high yield potential and low sulphur requirement. The sulphur requirement of cereals to produce one ton of cereals is low but uptake per unit area becomes almost equal to that of oilseeds mainly due to higher productivity of cereals (Sutar et al., 2017). Sulphur is an essential nutrient for all organisms due to its function in a large variety of processes (Gangadhara et al., 1990).

In the early 1990's, sulphur deficiencies in Indian soils were estimated to occur in about 130 districts. More recently, soil fertility surveys by the ICAR system (analysis of 60,000 soil samples 2009) have shown sulphur deficiencies to be a wide spread problem. A soil is considered deficient in $\mathrm{S}$ if it tests less than $10 \mathrm{mg} \mathrm{S} / \mathrm{kg}$ soil extractable with $0.15 \%$ $\mathrm{CaCl}_{2}$. Sulphur status of Indian soils is going down with each passing year. Close to $70 \%$ of soil samples analyzed by the ICAR system and TSI-FAI-IFA (The Sulphur Institute) project and other programs have been found to be either deficient or marginal in plant available sulphur. Soil analysis and crop response data generated by the TSI-FAI-IFA project (1997-2006) re-enforced the findings of the ICAR system. Based on reported results, out of over 49,000 soil samples 
analyzed across 18 states, $46 \%$ of samples were deficient in sulphur and another 30\% were medium in available sulphur which could be considered as potentially sulphur deficient. These data prove that sulphur deficiencies are a critical problem in $40-45 \%$ of districts translating into 57-64 million ha of net sown area.

\section{Materials and Methods}

A field experiment was carried out during kharif 2017 at College Farm, College of Agriculture, Rajendranagar, Hyderabad which is geographically situated at $17^{\circ} 19^{\circ} \mathrm{N}$ latitude and $78^{\circ} 23^{\circ} \mathrm{E}$ longitude and an altitude of $542.3 \mathrm{~m}$ above mean sea level. It is in the Southern Telangana agro-climatic zone of Telangana. According to Troll's climatic classification, it falls under semi-arid tropics (SAT). The experimental site was sandy loam in texture with $\mathrm{pH}(608), \mathrm{EC}\left(0.28 \mathrm{dSm}^{-1}\right)$, and OC (Low).The soil was low in available nitrogen $\left(120 \mathrm{~kg} \mathrm{ha}^{-1}\right)$ and available sulphur $\left(21.2 \mathrm{~kg} \mathrm{ha}^{-1}\right)$ and high in available phosphorus $\left(74 \mathrm{~kg} \mathrm{ha}^{-1}\right)$ and available potassium $(308 \mathrm{~kg}$ $\left.\mathrm{ha}^{-1}\right)$. Bulk density, porosity and moisture holding capacity were also determined by Keen's cup method (initial and final). The experiment was carried out with three different sources of sulphur (Gypsum, Ammonium Sulphate and Bentonite Sulphur) and three levels (S@40 kg ha ${ }^{-1}, \mathrm{~S} @ 60 \mathrm{~kg}$ $\mathrm{ha}^{-1}$ and $\mathrm{S} @ 80 \mathrm{~kg} \mathrm{ha}^{-1}$ ) and one source as urea, DAP and MOP and another source as urea, SSP and MOP comprising of eleven treatment combinations which were laid out in randomized block design and replicated thrice.

In all the treatments nitrogen was applied in two split doses $50 \%$ as basal and $50 \%$ as top dressing 45 DAS and entire dose of phosphorous, potassium and sulphur were applied as basal. Treatment $\mathrm{T}_{1}$ was application of recommended dose of fertilizer i.e., N$\mathrm{P}_{2} \mathrm{O}_{5}-\mathrm{K}_{2} \mathrm{O}-\mathrm{S} @$ 200-60-50-60 kg ha ${ }^{-1}$ without sulphur. Treatment $T_{2}$ was application of recommended dose of fertilizer through Urea, SSP and MOP. $\mathrm{T}_{3}$ was $\mathrm{T}_{1}+$ application of sulphur@ $90 \mathrm{~kg} \mathrm{ha}^{-1}$ through gypsum; $\mathrm{T}_{4}$ was $\mathrm{T}_{1}+$ application of sulphur @ $60 \mathrm{~kg} \mathrm{ha}^{-1}$ through gypsum; $\mathrm{T}_{5}$ was $\mathrm{T}_{1}+$ application of sulphur@80 kg ha ${ }^{-1}$ through gypsum. $\mathrm{T}_{6}$ was $\mathrm{T}_{1}+$ application of sulphur @ $40 \mathrm{~kg} \mathrm{ha}^{-1}$ through ammonium sulphate. $\mathrm{T}_{7}$ was $\mathrm{T}_{1}+$ application of sulphur @ $60 \mathrm{~kg} \mathrm{ha}^{-1}$ through ammonium sulphate. $\mathrm{T}_{8}$ was $\mathrm{T}_{1}+$ application of sulphur@80 kg ha ${ }^{-1}$ through ammonium sulphate. $\mathrm{T}_{9}$ was $\mathrm{T}_{1}+$ application of sulphur @ $40 \mathrm{~kg} \mathrm{ha}^{-1}$ through bentonite sulphur. $\mathrm{T}_{10}$ was $\mathrm{T}_{1}+$ application of sulphur @ $60 \mathrm{~kg} \mathrm{ha}^{-1}$ through bentonite sulphur. $\mathrm{T}_{10}$ was $\mathrm{T}_{1}+$ application of sulphur @ $80 \mathrm{~kg} \mathrm{ha}^{-1}$ through bentonite sulphur. Initial (30 DAS) and final (harvest) plant population was recorded. Plant samples were collected from gross plot to record dry matter production, plant height and LAI. To determine grain yield cobs from net plot were collected, sundried till $12 \%$ moisture attained and weighed. To determine stover yield stalk were harvested to ground level and sundried.

\section{Results and Discussion}

Initial and final plant population was taken at 30 DAS and harvest and was found nonsignificant when analysed statistically among the treatments. Plant height was nonsignificant at 30 DAS but at 60 DAS, 90 DAS and harvest $T_{2}$ and $T_{10}$ recorded highest values which were on par followed by $\mathrm{T}_{4}$ and $\mathrm{T}_{7}$. Lowest plant height was recorded by $\mathrm{T}_{1}$ (Table 1). Different sources and levels of sulphur were non-significant with respect to crop phenology. Different sources and levels of sulphur showed significant influence on LAI (Table 2) and TDM (Table 3) at 30 DAS, 60 DAS, 90 DAS and harvest, $\mathrm{T}_{2}$ and $\mathrm{T}_{10}$ recorded highest LAI and TDM values which were on par followed by $\mathrm{T}_{4}$ and $\mathrm{T}_{7}$. Least LAI and TDM was achieved by $\mathrm{T}_{1}$. 
Table.1 Plant height of maize (cms) at different stages as influenced by different sources and levels of Sulphur

\begin{tabular}{|c|c|c|c|c|c|}
\hline \multicolumn{2}{|r|}{ Treatments } & 30 & 60 DAS & 90 & $\begin{array}{l}\text { Harve } \\
\text { st }\end{array}$ \\
\hline$T_{1}$ & $\begin{array}{l}\text { Application of recommended dose of NPK through } \\
\text { Urea, DAP and MOP }\end{array}$ & 50.4 & 128.4 & 144.7 & 144.7 \\
\hline $\mathbf{T}_{2}$ & $\begin{array}{l}\text { Application of recommended dose of NPK through } \\
\text { Urea, SSP and MOP (sulphur supplied through SSP is } \\
41.25 \mathrm{~kg} \mathrm{ha}^{-1)}\end{array}$ & 51.3 & 134.1 & 150.6 & 150.4 \\
\hline$T_{3}$ & $\begin{array}{l}\mathrm{T}_{1}+\text { Application of Sulphur @ } 40 \mathrm{~kg} \mathrm{ha}^{-1} \text { through } \\
\text { gypsum }\end{array}$ & 50.8 & 132.6 & 149.0 & 149.0 \\
\hline$T_{4}$ & $\begin{array}{l}\mathrm{T}_{1}+\text { Application of Sulphur @ } 60 \mathrm{~kg} \mathrm{ha}^{-1} \text { through } \\
\text { gypsum }\end{array}$ & 50.6 & 133.9 & 150.3 & 150.3 \\
\hline$T_{5}$ & $\begin{array}{l}\mathrm{T}_{1}+\text { Application of Sulphur @ } 80 \mathrm{~kg} \mathrm{ha}^{-1} \text { through } \\
\text { gypsum }\end{array}$ & 51.0 & 133.6 & 149.9 & 149.9 \\
\hline$T_{6}$ & $\begin{array}{l}\mathrm{T}_{1}+\text { Application of Sulphur @ } 40 \mathrm{~kg} \mathrm{ha}^{-1} \text { through } \\
\text { Ammonium Sulphate }\end{array}$ & 50.7 & 132.4 & 148.9 & 148.9 \\
\hline $\mathbf{T}_{7}$ & $\begin{array}{l}\mathrm{T}_{1}+\text { Application of Sulphur @ } 60 \mathrm{~kg} \mathrm{ha}^{-1} \text { through } \\
\text { Ammonium Sulphate }\end{array}$ & 50.5 & 133.9 & 150.3 & 150.3 \\
\hline$T_{8}$ & $\begin{array}{l}\mathrm{T}_{1}+\text { Application of Sulphur @ } 80 \mathrm{~kg} \mathrm{ha}^{-1} \text { through } \\
\text { Ammonium Sulphate }\end{array}$ & 50.7 & 133.5 & 150.1 & 150.1 \\
\hline$T_{9}$ & $\begin{array}{l}\mathrm{T}_{1}+\text { Application of Sulphur @ } 40 \mathrm{~kg} \mathrm{ha}^{-1} \text { through } \\
\text { Bentonite Sulphur }\end{array}$ & 50.4 & 131.7 & 148.0 & 148.0 \\
\hline$T_{10}$ & $\begin{array}{l}\mathrm{T}_{1}+\text { Application of Sulphur @ } 60 \mathrm{~kg} \mathrm{ha}^{-1} \text { through } \\
\text { Bentonite Sulphur }\end{array}$ & 51.7 & 134.6 & 151.0 & 151.0 \\
\hline$T_{11}$ & $\begin{array}{l}\mathrm{T}_{1}+\text { Application of Sulphur @ } 80 \mathrm{~kg} \mathrm{ha}^{-1} \text { through } \\
\text { Bentonite Sulphur }\end{array}$ & 50.9 & 133.8 & 150.3 & 150.3 \\
\hline & S. $E(m) \pm$ & 0.64 & 0.55 & 0.55 & 0.55 \\
\hline & $\mathrm{CD}(5 \%)$ & NS & 1.15 & 2.17 & 2.17 \\
\hline
\end{tabular}


Table.2 LAI of maize at different stages as influenced by different sources and levels of Sulphur

\begin{tabular}{|c|c|c|c|c|c|}
\hline \multicolumn{2}{|r|}{ Treatments } & & & & Harves \\
\hline $\mathbf{T}_{1}$ & $\begin{array}{l}\text { Application of recommended dose of NPK through } \\
\text { Urea, DAP and MOP }\end{array}$ & 0.32 & 5.8 & 4.6 & 3.6 \\
\hline $\mathbf{T}_{2}$ & $\begin{array}{l}\text { Application of recommended dose of NPK through } \\
\text { Urea, SSP and MOP (sulphur supplied through SSP is } \\
41.25 \mathrm{~kg} \mathrm{ha}^{-1)}\end{array}$ & 0.40 & 6.4 & 5.2 & 4.2 \\
\hline $\mathbf{T}_{3}$ & $\begin{array}{l}\mathrm{T}_{1}+\text { Application of Sulphur @ } 40 \mathrm{~kg} \mathrm{ha}^{-1} \text { through } \\
\text { gypsum }\end{array}$ & 0.38 & 6.0 & 4.8 & 3.9 \\
\hline $\mathbf{T}_{4}$ & $\begin{array}{l}\mathrm{T}_{1}+\text { Application of Sulphur @ } 60 \mathrm{~kg} \mathrm{ha}^{-1} \text { through } \\
\text { gypsum }\end{array}$ & 0.40 & 6.3 & 5.1 & 4.2 \\
\hline $\mathbf{T}_{5}$ & $\begin{array}{l}\mathrm{T}_{1}+\text { Application of Sulphur @ } 80 \mathrm{~kg} \mathrm{ha}^{-1} \text { through } \\
\text { gypsum }\end{array}$ & 0.40 & 6.3 & 5.1 & 4.1 \\
\hline$T_{6}$ & $\begin{array}{l}\mathrm{T}_{1}+\text { Application of Sulphur @ } 40 \mathrm{~kg} \mathrm{ha}^{-1} \text { through } \\
\text { Ammonium Sulphate }\end{array}$ & 0.38 & 6.0 & 4.7 & 3.9 \\
\hline $\mathbf{T}_{7}$ & $\begin{array}{l}\mathrm{T}_{1}+\text { Application of Sulphur @ } 60 \mathrm{~kg} \mathrm{ha}^{-1} \text { through } \\
\text { Ammonium Sulphate }\end{array}$ & 0.40 & 6.3 & 5.1 & 4.2 \\
\hline $\mathbf{T}_{8}$ & $\begin{array}{l}\mathrm{T}_{1}+\text { Application of Sulphur @ } 80 \mathrm{~kg} \mathrm{ha}^{-1} \text { through } \\
\text { Ammonium Sulphate }\end{array}$ & 0.40 & 6.3 & 5.1 & 4.1 \\
\hline$T_{9}$ & $\begin{array}{l}\mathrm{T}_{1}+\text { Application of Sulphur @ } 40 \mathrm{~kg} \mathrm{ha}^{-1} \text { through } \\
\text { Bentonite Sulphur }\end{array}$ & 0.38 & 6.0 & 4.8 & 3.9 \\
\hline $\mathbf{T}_{10}$ & $\begin{array}{l}\mathrm{T}_{1}+\text { Application of Sulphur @ } 60 \mathrm{~kg} \mathrm{ha}^{-1} \text { through } \\
\text { Bentonite Sulphur }\end{array}$ & 0.41 & 6.5 & 5.2 & 4.2 \\
\hline $\mathbf{T}_{11}$ & $\begin{array}{l}\mathrm{T}_{1}+\text { Application of Sulphur @ } 80 \mathrm{~kg} \mathrm{ha}^{-1} \text { through } \\
\text { Bentonite Sulphur }\end{array}$ & 0.39 & 6.3 & 5.0 & 4.1 \\
\hline & S. E $(\mathrm{m}) \pm$ & 0.01 & 0.03 & 0.02 & 0.06 \\
\hline & $\mathrm{CD}(5 \%)$ & 0.02 & 0.45 & 0.36 & 0.13 \\
\hline
\end{tabular}


Table.3 Dry matter production $\left(\mathrm{g} \mathrm{plant}^{-1}\right.$ )of maize at different stages as influenced by different sources and levels of sulphur

\begin{tabular}{|c|c|c|c|c|c|}
\hline \multicolumn{2}{|r|}{ Treatments } & 30 DAS & 60 DAS & 90 DAS & Harvest \\
\hline $\mathbf{T}_{1}$ & $\begin{array}{l}\text { Application of recommended } \\
\text { dose of NPK through Urea, } \\
\text { DAP \& MOP }\end{array}$ & 10.2 & 80.0 & 126.2 & 138.2 \\
\hline $\mathbf{T}_{2}$ & $\begin{array}{l}\text { Application of recommended } \\
\text { dose of NPK through Urea, } \\
\text { SSP \& MOP (sulphur supplied } \\
\text { through SSP is } 41.25 \mathrm{~kg} \mathrm{ha}^{-1)}\end{array}$ & 13.3 & 89.3 & 129.3 & 141.3 \\
\hline $\mathbf{T}_{3}$ & $\begin{array}{l}\mathrm{T}_{1}+\text { Application of Sulphur @ } \\
40 \mathrm{~kg} \mathrm{ha}^{-1} \text { through gypsum }\end{array}$ & 10.3 & 85.0 & 126.3 & 138.3 \\
\hline $\mathbf{T}_{4}$ & $\begin{array}{l}\mathrm{T}_{1}+\text { Application of Sulphur @ } \\
60 \mathrm{~kg} \mathrm{ha}^{-1} \text { through gypsum }\end{array}$ & 12.3 & 87.3 & 128.3 & 140.3 \\
\hline $\mathbf{T}_{5}$ & $\begin{array}{l}\mathrm{T}_{1}+\text { Application of Sulphur @ } \\
80 \mathrm{~kg} \mathrm{ha}^{-1} \text { through gypsum }\end{array}$ & 12.3 & 81.3 & 128.3 & 140.3 \\
\hline $\mathbf{T}_{6}$ & $\begin{array}{l}\mathrm{T}_{1}+\text { Application of Sulphur @ } \\
40 \mathrm{~kg} \mathrm{ha}^{-1} \text { through } \\
\text { Ammonium Sulphate }\end{array}$ & 10.3 & 87.7 & 126.3 & 138.3 \\
\hline $\mathbf{T}_{7}$ & $\begin{array}{l}\mathrm{T}_{1}+\text { Application of Sulphur @ } \\
60 \mathrm{~kg} \mathrm{ha}^{-1} \text { through } \\
\text { Ammonium Sulphate }\end{array}$ & 12.7 & 88.7 & 128.7 & 140.7 \\
\hline $\mathrm{T}_{8}$ & $\begin{array}{l}\mathrm{T}_{1}+\text { Application of Sulphur @ } \\
80 \mathrm{~kg} \mathrm{ha}^{-1} \text { through } \\
\text { Ammonium Sulphate }\end{array}$ & 12.3 & 86.7 & 128.3 & 140.3 \\
\hline $\mathbf{T}_{9}$ & $\begin{array}{l}\mathrm{T}_{1}+\text { Application of Sulphur @ } \\
40 \mathrm{~kg} \mathrm{ha}^{-1} \text { through Bentonite } \\
\text { Sulphur }\end{array}$ & 10.7 & 82.7 & 126.7 & 138.7 \\
\hline $\mathbf{T}_{10}$ & $\begin{array}{l}\mathrm{T}_{1}+\text { Application of Sulphur } \\
@ 60 \mathrm{~kg} \mathrm{ha}^{-1} \text { through } \\
\text { Bentonite Sulphur }\end{array}$ & 13.3 & 89.3 & 129.3 & 141.3 \\
\hline$T_{11}$ & $\begin{array}{l}\mathrm{T}_{1}+\text { Application of Sulphur @ } \\
80 \mathrm{~kg} \mathrm{ha}^{-1} \text { through Bentonite } \\
\text { Sulphur }\end{array}$ & 11.6 & 88.3 & 127.6 & 139.6 \\
\hline & S. Em. +/- & 0.48 & 1.04 & 1.02 & 1.02 \\
\hline & $\mathrm{CD}(5 \%)$ & 1.00 & 2.17 & 1.17 & 1.17 \\
\hline
\end{tabular}




\begin{tabular}{|c|c|c|c|c|c|}
\hline \multicolumn{2}{|r|}{ Treatments } & \multirow{2}{*}{$\begin{array}{l}\begin{array}{l}\text { No of } \\
\text { cobs } \\
\text { plant-1 }\end{array} \\
1.0\end{array}$} & \multirow{2}{*}{$\begin{array}{l}\text { No. of } \\
\text { rows } \\
\text { cob }^{-1} \\
12.0\end{array}$} & \multirow{2}{*}{$\begin{array}{l}\text { No. of } \\
\text { grains } \\
\text { row }^{-1} \\
20.7\end{array}$} & \multirow{2}{*}{$\begin{array}{l}\text { No of grains } \\
\text { cob }^{-1}\end{array}$} \\
\hline $\mathbf{T}_{1}$ & $\begin{array}{l}\text { Application of recommended } \\
\text { dose of NPK through Urea, } \\
\text { DAP and MOP }\end{array}$ & & & & \\
\hline $\mathbf{T}_{2}$ & $\begin{array}{l}\text { Application of recommended } \\
\text { dose of NPK through Urea, } \\
\text { SSP and MOP (sulphur } \\
\text { supplied through SSP is } \\
41.25 \mathrm{~kg} \mathrm{ha}^{-1)}\end{array}$ & 1.0 & 13.3 & 23.7 & 306.0 \\
\hline $\mathbf{T}_{3}$ & $\begin{array}{l}\mathrm{T}_{1}+\text { Application of Sulphur } \\
\text { @ } 40 \mathrm{~kg} \mathrm{ha}^{-1} \text { through gypsum }\end{array}$ & 1.0 & 13.3 & 22.3 & 277.3 \\
\hline $\mathbf{T}_{4}$ & $\begin{array}{l}\mathrm{T}_{1}+\text { Application of Sulphur } \\
@ 60 \mathrm{~kg} \mathrm{ha}^{-1} \text { through gypsum }\end{array}$ & 1.0 & 13.3 & 22.7 & 302.7 \\
\hline $\mathbf{T}_{5}$ & $\begin{array}{l}\mathrm{T}_{1}+\text { Application of Sulphur } \\
\text { @ } 80 \mathrm{~kg} \mathrm{ha}^{-1} \text { through gypsum }^{-}\end{array}$ & 1.0 & 12.7 & 21.3 & 284 \\
\hline $\mathbf{T}_{6}$ & $\begin{array}{l}\mathrm{T}_{1}+\text { Application of Sulphur } \\
\text { @ } 40 \mathrm{~kg} \mathrm{ha}^{-1} \text { through } \\
\text { Ammonium Sulphate }\end{array}$ & 1.0 & 13.3 & 22.3 & 281.3 \\
\hline $\mathbf{T}_{7}$ & $\begin{array}{l}\mathrm{T}_{1}+\text { Application of Sulphur } \\
\text { @ } 60 \mathrm{~kg} \mathrm{ha}^{-1} \text { through } \\
\text { Ammonium Sulphate }\end{array}$ & 1.0 & 13.3 & 22.8 & 302.7 \\
\hline $\mathbf{T}_{8}$ & $\begin{array}{l}\mathrm{T}_{1}+\text { Application of Sulphur } \\
@ 80 \mathrm{~kg} \mathrm{ha}^{-1} \text { through } \\
\text { Ammonium Sulphate }\end{array}$ & 1.0 & 12.7 & 22.6 & 286.7 \\
\hline$T_{9}$ & $\begin{array}{l}\mathrm{T}_{1}+\text { Application of Sulphur } \\
\text { @ } 40 \mathrm{~kg} \mathrm{ha}^{-1} \text { through } \\
\text { Bentonite Sulphur }\end{array}$ & 1.0 & 13.3 & 22.3 & 282.0 \\
\hline $\mathbf{T}_{10}$ & $\begin{array}{l}\mathrm{T}_{1}+\text { Application of Sulphur } \\
\text { @ } 60 \mathrm{~kg} \mathrm{ha}^{-1} \text { through } \\
\text { Bentonite Sulphur }\end{array}$ & 1.0 & 13.3 & 24.0 & 320.0 \\
\hline $\mathbf{T}_{11}$ & $\begin{array}{l}\mathrm{T}_{1}+\text { Application of Sulphur } \\
\text { @ } 80 \mathrm{~kg} \mathrm{ha}^{-1} \text { through } \\
\text { Bentonite Sulphur }\end{array}$ & 1.0 & 12.7 & 22.8 & 294.0 \\
\hline & S. $E(m) \pm$ & 0.0 & 0.7 & 0.7 & 18.1 \\
\hline & $\mathrm{CD}(5 \%)$ & NS & NS & 1.4 & 37.9 \\
\hline
\end{tabular}




\begin{tabular}{|l|l|l|l|}
\hline \multicolumn{5}{|c|}{ Table.5 Test weight $(\mathrm{g})$, grain yield $\left(\mathrm{kg} \mathrm{ha}{ }^{-1}\right)$ and stover yield $\left(\mathrm{kg} \mathrm{ha}^{-1)}\right.$} \\
influenced by different sources and levels of Sulphur
\end{tabular}


Table.6 Sulphur uptake ( $\mathrm{kg} \mathrm{ha}^{-1}$ ) of maize in stover and seed as influenced by different sources and levels of Sulphur

\begin{tabular}{|c|c|c|c|c|}
\hline \multirow{2}{*}{\multicolumn{2}{|c|}{ Treatments }} & \multicolumn{2}{|c|}{ Sulphur } & \multirow{2}{*}{$\begin{array}{l}\text { Sulphur uptake } \\
\text { in stover and } \\
\text { seed }\end{array}$} \\
\hline & & \multirow{2}{*}{$\begin{array}{l}\text { Stover } \\
12.15\end{array}$} & \multirow{2}{*}{$\begin{array}{l}\text { seed } \\
5.67\end{array}$} & \\
\hline$T_{1}$ & $\begin{array}{l}\text { Application of recommended dose of } \\
\text { NPK through Urea, DAP and MOP }\end{array}$ & & & 17.82 \\
\hline $\mathbf{T}_{2}$ & 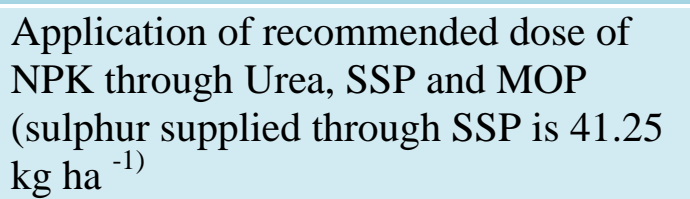 & 12.88 & 10.24 & 23.12 \\
\hline$T_{3}$ & $\begin{array}{l}\mathrm{T}_{1}+\text { Application of Sulphur @ } 40 \mathrm{~kg} \mathrm{ha}^{-} \\
{ }^{1} \text { through gypsum }\end{array}$ & 13.21 & 10.03 & 23.24 \\
\hline $\mathbf{T}_{4}$ & $\begin{array}{l}\mathrm{T}_{1}+\text { Application of Sulphur @ } 60 \mathrm{~kg} \mathrm{ha}^{-} \\
{ }^{1} \text { through gypsum }\end{array}$ & 16.34 & 14.25 & 30.59 \\
\hline $\mathbf{T}_{5}$ & $\begin{array}{l}\mathrm{T}_{1}+\text { Application of Sulphur @ } 80 \mathrm{~kg} \mathrm{ha}^{-} \\
{ }^{1} \text { through gypsum }\end{array}$ & 15.90 & 13.26 & 29.16 \\
\hline$T_{6}$ & $\begin{array}{l}\mathrm{T}_{1}+\text { Application of Sulphur @ } 40 \mathrm{~kg} \mathrm{ha}^{-} \\
{ }^{1} \text { through Ammonium Sulphate }\end{array}$ & 13.44 & 10.26 & 23.70 \\
\hline $\mathbf{T}_{7}$ & $\begin{array}{l}\mathrm{T}_{1}+\text { Application of Sulphur @ } 60 \mathrm{~kg} \mathrm{ha}^{-} \\
{ }^{1} \text { through Ammonium Sulphate }\end{array}$ & 16.42 & 14.38 & 30.80 \\
\hline$T_{8}$ & $\begin{array}{l}\mathrm{T}_{1}+\text { Application of Sulphur @ } 80 \mathrm{~kg} \mathrm{ha} \\
{ }^{1} \text { through Ammonium Sulphate }\end{array}$ & 15.86 & 13.31 & 29.17 \\
\hline$T_{9}$ & $\begin{array}{l}\mathrm{T}_{1}+\text { Application of Sulphur @ } 40 \mathrm{~kg} \mathrm{ha}^{-} \\
{ }^{1} \text { through Bentonite Sulphur }\end{array}$ & 13.54 & 10.37 & 23.91 \\
\hline $\mathbf{T}_{10}$ & $\begin{array}{l}\mathrm{T}+\text { Application of Sulphur @ } 60 \mathrm{~kg} \\
\mathrm{ha}^{-1} \text { through Bentonite Sulphur }\end{array}$ & 16.46 & 14.83 & 31.29 \\
\hline $\mathbf{T}_{11}$ & $\begin{array}{l}\mathrm{T}_{1}+\text { Application of Sulphur @ } 80 \mathrm{~kg} \mathrm{ha} \\
{ }^{1} \text { through Bentonite Sulphur }\end{array}$ & 15.96 & 13.69 & 29.65 \\
\hline & S. E $(\mathrm{m})+$ & 0.29 & 0.14 & 0.33 \\
\hline & $\mathrm{CD}(5 \%)$ & 0.61 & 0.29 & 0.69 \\
\hline
\end{tabular}


Table.7 Effect of different sources and levels of sulphur on economics

\begin{tabular}{|c|c|c|c|c|c|}
\hline \multicolumn{2}{|c|}{ Treatments } & \multirow{2}{*}{$\begin{array}{l}\begin{array}{l}\text { Cost of } \\
\text { Cultivation } \\
\left.\left(\text { Rs. }^{-1}\right)^{-1}\right)\end{array} \\
22936.00\end{array}$} & \multirow{2}{*}{$\begin{array}{l}\text { Gross } \\
\text { returns( } \\
\left.\text { Rs. ha }^{-1}\right) \\
77176.65\end{array}$} & \multirow{2}{*}{$\begin{array}{l}\begin{array}{l}\text { Net } \\
\text { returns(R } \\
\left.\text { s.ha }^{-1}\right)\end{array} \\
50109.30\end{array}$} & \multirow{2}{*}{$\begin{array}{l}\text { B:C } \\
\text { ratio } \\
2.59 \\
\end{array}$} \\
\hline $\mathrm{T}_{1}$ & $\begin{array}{l}\text { Application of recommended dose of } \\
\text { NPK through Urea, DAP and MOP }\end{array}$ & & & & \\
\hline $\mathbf{T}_{2}$ & $\begin{array}{l}\text { Application of recommended dose of } \\
\text { NPK through Urea, SSP and MOP } \\
\text { (sulphur supplied through SSP is } 41.25 \\
\mathrm{~kg} \mathrm{ha}^{-1)}\end{array}$ & 22869.57 & 78623.80 & 52154.23 & 3.04 \\
\hline$T_{3}$ & $\begin{array}{l}\mathrm{T}_{1}+\text { Application of Sulphur @ } 40 \mathrm{~kg} \\
\text { ha }^{-1} \text { through gypsum }\end{array}$ & 23636.72 & 77499.80 & 50354.86 & 2.93 \\
\hline $\mathbf{T}_{4}$ & $\begin{array}{l}\mathrm{T}_{1}+\text { Application of Sulphur @ } 60 \mathrm{~kg} \mathrm{ha}^{-} \\
{ }^{-} \text {through gypsum }\end{array}$ & 23698.88 & 77537.27 & 49983.59 & 2.92 \\
\hline $\mathbf{T}_{5}$ & $\begin{array}{l}\mathrm{T}_{1}+\text { Application of Sulphur @ } 80 \mathrm{~kg} \\
\text { ha }^{-1} \text { through gypsum }\end{array}$ & 24118.88 & 77452.97 & 48962.73 & 2.87 \\
\hline$T_{6}$ & $\begin{array}{l}\mathrm{T}_{1}+\text { Application of Sulphur @ } 40 \mathrm{~kg} \\
\mathrm{ha}^{-1} \text { through Ammonium Sulphate }\end{array}$ & 24677.98 & 77518.53 & 50239.65 & 2.82 \\
\hline $\mathbf{T}_{7}$ & $\begin{array}{l}\mathrm{T}_{1}+\text { Application of Sulphur @ } 60 \mathrm{~kg} \\
\mathrm{ha}^{-1} \text { through Ammonium Sulphate }\end{array}$ & 25588.46 & 77527.90 & 49829.02 & 2.75 \\
\hline $\mathrm{T}_{8}$ & $\begin{array}{l}\mathrm{T}_{1}+\text { Application of Sulphur @ } 80 \mathrm{~kg} \\
\text { ha }^{-1} \text { through Ammonium Sulphate }\end{array}$ & 26522.80 & 77649.66 & 49530.79 & 2.64 \\
\hline$T_{9}$ & $\begin{array}{l}\mathrm{T}_{1}+\text { Application of Sulphur @ } 40 \mathrm{~kg} \\
\text { ha }^{-1} \text { through Bentonite Sulphur }\end{array}$ & 25452.00 & 79729.07 & 51171.83 & 2.75 \\
\hline$T_{10}$ & $\begin{array}{l}\mathrm{T}_{1}+\text { Application of Sulphur @ } 60 \mathrm{~kg} \\
\text { ha }^{-1} \text { through Bentonite Sulphur }\end{array}$ & 26178.00 & 80139.34 & 50236.60 & 2.71 \\
\hline $\mathbf{T}_{11}$ & $\begin{array}{l}\mathrm{T}_{1}+\text { Application of Sulphur @ } 80 \mathrm{~kg} \\
\text { ha }^{-1} \text { through Bentonite Sulphur }\end{array}$ & 27348.00 & 79897.66 & 48707.94 & 2.57 \\
\hline & S. E $(m) \pm$ & & 275.00 & 274.00 & 0.01 \\
\hline & $\mathrm{CD}(5 \%)$ & & 818.00 & 813.98 & 0.02 \\
\hline
\end{tabular}


Yield attributes i.e., number of cobs plant ${ }^{-1}$ and number of rows $\operatorname{cob}^{-1}$ were nonsignificant when analysed statistically but number of grains row ${ }^{-1}$ and number of grains $\operatorname{cob}^{-1}$ were significant among different treatments, $T_{2}$ and $T_{10}$ recorded highest values which were on par followed by $T_{4}$ and $T_{7}$. Lowest data was recorded by $\mathrm{T}_{1}$ (Table 4 ).

Highest grain (5596 kg ha ${ }^{-1}$ ) and stover yield $\left(6995 \mathrm{~kg} \mathrm{ha}^{-1}\right)$ was recorded by treatment $\mathrm{T}_{10}$ (S@60 kg ha- ${ }^{-1}$ through bentonite sulphur) followed by treatment $\mathrm{T}_{2}$ (5581 and $6976 \mathrm{~kg}$ ha $^{-1}$ ) S@40 kg ha ${ }^{-1}$ through urea, SSP and DAP. Treatments $\mathrm{T}_{7}$ and $\mathrm{T}_{4}$ were statistically on par with each other. Similarly treatments $\mathrm{T}_{3}, \mathrm{~T}_{6}$ and $\mathrm{T}_{9}$ were statistically on par with each other followed by $\mathrm{T}_{5}, \mathrm{~T}_{9}$ and $\mathrm{T}_{11}$ which were in turn on par (Table 5). Lowest grain $\left(5477 \mathrm{~kg} \mathrm{ha}^{-1}\right)$ and stover yield (6847 $\left.\mathrm{kg} \mathrm{ha}^{-1}\right)$ was recorded by $T_{1}$ which was non sulphur treatment.

Initial and final soil physico chemical properties like bulk density, porosity, maximum water holding capacity, soil texture, $\mathrm{pH}, \mathrm{Ec}, \mathrm{OC}$ were found nonsignificant when analysed statistically. Initial and final nutrient status of soil was also nonsignificant.

Highest sulphur uptake in stover and seed was noted by $\mathrm{T}_{10}$ and lowest by $\mathrm{T}_{1}$ (Table 6 ).

Highest gross returns were recorded by $T_{10}$ (Rs. $80139 \mathrm{ha}^{-1}$ ) followed by $\mathrm{T}_{2}$ (Rs. 78623 ha $^{-1}$ ) though highest $\mathrm{B}$ : $\mathrm{C}$ ratio was recorded by $\mathrm{T}_{2}$ because of higher cost of bentonite sulphur fertilizer (Table 7).

Highest growth and yield parameters were recorded by treatment $\mathrm{T}_{11}$ followed by treatment $\mathrm{T}_{2}$, but highest $\mathrm{B}$ : $\mathrm{C}$ ratio was recorded by treatment $T_{2}$. Variation in soil physico chemical properties was nonsignificant among different treatments but sulphur uptake studies indicate that uptake was highest at $60 \mathrm{~kg} \mathrm{ha}^{-1}$ and $80 \mathrm{~kg} \mathrm{ha}^{-1}$ which were statistically on par.

\section{Acknowledgements}

My earnest acknowledgement goes to my guides Dr. G. E. Ch. Vidyasagar, Dr. P. Suresh and Dr. S. Harish Kumar Sharma for their scholastic guidance, valuable knowledge and technical advice. I than all my teachers, friends and university for their valuable cooperation and help during my research program.

\section{References}

Abbas, G., Hussain, A., Ahmad, A and Wajid, A. S. 2005. Effect of irrigation schedules and nitrogen rates on yield and yield components of maize. Journal of agricultural and social sciences. 1 (4): 335-338.

Binod Kumar, Govind Singh, Rajesh Kumar and Kamlesh Kumar Nishad.2016. Effect of nitrogen and sulphur nutrition on growth and yield of maize (Zea mays L.) under eastern plain zone of U.P. Intl.J.Agric.Sci., 12(2): 181-185.

Channabasamma, A., Habsur, N. S., Bangaremma, S. W and Akshaya, M. C. 2013. Effect of nitrogen and sulphur levels and ratios on growth and yield of maize. Molecular Plant Breeding. 37 (4): 292-296.

Gangadhara, G. A., Manjunathaiah, H. M and Satyanarayana, T. 1990. Effect of sulphur on yield, oil content of sunflower and uptake of micronutrients by plants. Journal of Indian Society of Soil Science. 38(4): 692-694.

Krishnamoorthy, S. K. 1989. Sulphur fertilization for yield and quality of crops. Proc. National seminar on Sulphur in Agriculture, held at UAS, Bangalore. Pp 22-25. 
Kumar, H. and Yadav, D. S. 2007. Effect of phosphorus and sulphur level on growth, yield and quality of Indian mustard (Brassica juncia L.) cultivars. Indian Journal of Agronomy. 52(2): 154-157.

Marschner, H. 1995. Mineral Nutrition of Higher Plants $\left(2^{\text {nd }}\right.$ Ed.). Academic Press, London.

Rahul K. Sutar, Amit M. Pujar, Aravinda Kumar B.N. and Hebsur N.S. 2017.
Sulphur nutrition in maize a critical review. Int. J. Pure App. Biosci. 5(6): 1582-1596.

Saalbach, E. 1973. The effect of S, Mg and $\mathrm{Na}$ on yield and quality of agriculture crop. Pontificial Academical Scientiarum Scripa Varia. 38: 451-538. Tisdale, S. L., Nelson, W. L and Beaton, J. D. 1985. Soil Fertility and Fertilizers; Macmillan Publishing Company: New York, 75-79.

\section{How to cite this article:}

Padma, P.V., G.E.CH. Vidyasagar, P. Suresh and Harish Kumar Sharma, S. 2018. Effect of Different Sources and Levels of Sulphur on Growth and Yield of Maize (Zea mays L.). Int.J.Curr.Microbiol.App.Sci. 7(08): 1548-1559. doi: https://doi.org/10.20546/ijcmas.2018.708.177 